\title{
Insights Into Regolith Evolution from TEM Studies of Space Weathering of Itokawa Particles.
}

\author{
Eve L. Berger ${ }^{1}$, Lindsay P. Keller ${ }^{2}$ \\ ${ }^{1}$ GeoControl Systems, Inc. - Jacobs JETS Contract - NASA Johnson Space Center, Houston, TX USA \\ ${ }^{2}$ NASA Johnson Space Center, Houston, TX USA
}

Exposure to solar wind irradiation and micrometeorite impacts alter the properties of regolith materials exposed on airless bodies [1]. However, estimates of space weathering rates for asteroid regoliths span many orders of magnitude [e.g., 2, 3]. Timescales for space weathering processes on airless bodies can be anchored by analyzing surface samples returned by JAXA's Hayabusa mission to asteroid 25143 Itokawa. Constraints on timescales of solar flare particle track accumulation $[4,5]$ and formation of solar wind produced ion-damaged rims [6] yield information on regolith dynamics.

Multiple electron transparent thin sections of Itokawa particles RAQD02-0211 (0211), RA-QD02-0125 (0125), and RA-QD02-0192 (0192) were prepared using a hybrid ultramicrotomy-focused ion beam (FIB) technique [7] on a Leica EM UC6 ultramicrotome and an FEI Quanta 3D dual beam FIB-SEM. This technique results in whole slices of particles - preserving both edge and interior features (e.g., Fig. 1a). Transmission electron microscope (TEM) analyses of the FIB sections, which allow for accurate determination of solar flare particle track densities and rim characteristics (e.g., width, crystallinity), were done on the JEOL 2500SE 200kV field emission STEM. All instruments are housed at NASA JSC.

All three particles are olivine-rich $\left(\mathrm{Fo}_{70}\right)$ with minor sulfides and show features of space weathering: adhering mineral grains and melt particles, solar flare particle tracks, and continuous solar wind damaged rims. The rims are compositionally similar to the cores of the grains, structurally disordered, and nanocrystalline [8]. The rim thickness varies and track density gradients (fig. 2) are observed across the particles. The track density gradient across particle 0211 correlates with the rim thickness (Figs. 1b). The highest track density $\left(3.4 \times 10^{9}\right.$ tracks $\left./ \mathrm{cm}^{2}\right)$ is on the side of the particle with the thickest rim $(\sim 80 \mathrm{~nm})$, while the lowest track density $\left(9.2 \times 10^{8}\right.$ tracks $\left./ \mathrm{cm}^{2}\right)$ correlates with the thinnest rim $(\sim 40 \mathrm{~nm})$. Particle 0192 also shows a track density gradient $\left(2.9 \times 10^{9}\right.$ to $1.1 \times 10^{9}$ tracks $\left./ \mathrm{cm}^{2}\right)$ and has comparable rim widths to particle 0211. Exposure ages, based on the track production rate of $4.1 \pm 1.2 \times 10^{4}$ tracks $/ \mathrm{cm}^{2} /$ year at $1 \mathrm{AU}[4]$ are: $\sim 80,000$ years for $0211, \sim 70,000$ years for 0192 , and $\sim 24,000$ years for 0125 .

The heterogeneous distribution of the space weathering effects on two Itokawa particles is consistent with both particles maintaining a relatively fixed orientation in the Itokawa regolith throughout the time they were being irradiated by incoming solar flare particles. 1.) Cosmic ray exposure ages for other Itokawa particles are relatively young $(\leq 1-1.5 \mathrm{Ma})$ [9-11] when compared to other LL chondrites (8-50Ma) [12]. The CRE age indicates that the regolith was stable at meter depths over $\sim 10^{6}$ years. 2.) Based on track production rates in olivine at $1 \mathrm{AU}$ [4], Itokawa particles recorded solar flare tracks over timescales of $<10^{5}$ years. The track gradient indicates that over this timescale, the particles maintained a relatively stable orientation at $\mathrm{mm}$ to $\mathrm{cm}$ depths. 3.) Solar wind produced ion damaged rims are predicted to become amorphous and to reach thicknesses of $100 \mathrm{~nm}$ within $10^{3}-10^{4}$ years [6]. The rims on the Itokawa particles are not amorphous and have thicknesses of $<60-70 \mathrm{~nm}$, suggesting a residence time on the surface (with direct exposure to the solar wind) of less than $\sim 10^{3}$ years. The continuous rims found on these grains, which have varying thicknesses, indicate that all sides of the 
particles have had direct exposure to the solar wind. The uppermost surface of the Itokawa regolith was sufficiently dynamic that while grain rotation must have occurred, the particles were not lost to space.

The Itokawa particles were shielded from direct exposure to the solar wind, at $\mathrm{mm}$ to $\mathrm{cm}$ depths, over timescales of $10^{4}-10^{5}$ years. The track gradients in these particles suggest that the regolith in the Muses$\mathrm{C}$ region of Itokawa experienced little overturn; rather, it was relatively stable at these depths. However, late in their history, over $<10^{3}$ years, all sides of the particles were directly exposed to the solar wind (as evidenced by continuous ion-damaged rims), which requires grain rotation on Itokawa's surface.

[1] B Hapke, Journal of Geophysical Research 106 (2001), p. 10039.

[2] M Willman et al., Icarus 208 (2010), p. 758.

[3] P Vernazza et al., Nature 458 (2009), p. 993.

[4] E L Berger and L P Keller, LPSC XLVI (2015), p. 1543.

[5] E L Berger and L P Keller, LPSC XLVI (2015), p. 2351.

[6] R Christoffersen and L P Keller, LPSC XLVI (2015), p. 2084

[7] E L Berger and L P Keller, Microscopy Today 23 (2015), p. 18.

[8] L P Keller and E L Berger, Earth, Planets \& Space 66 (2014), p. 71.

[9] M M M Meier et al., LPSC XLV (2014), p. 1247.

[10] K Nagao et al., LPSC XLIV (2013), p. 1976.

[11] K Nagao et al., Science 333 (2011), p. 1128.

[12] T Graf and K Marti, Meteoritics 29 (1994), p. 643
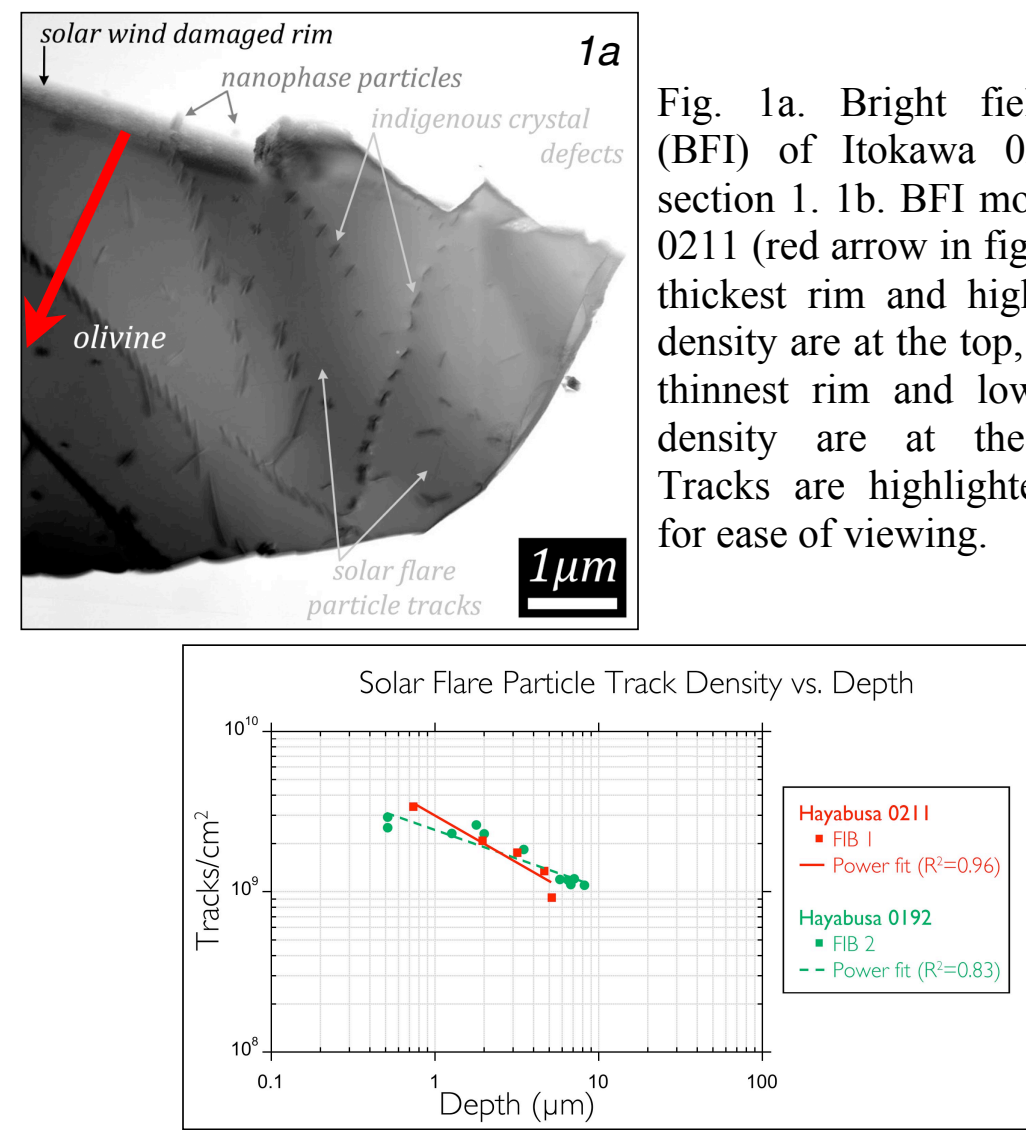

Fig. 2. Track density vs. depth. The trend line slopes are consistent with the Itokawa particles experiencing only mild tumbling while exposed on the surface of the asteroid; see $[4,5]$.

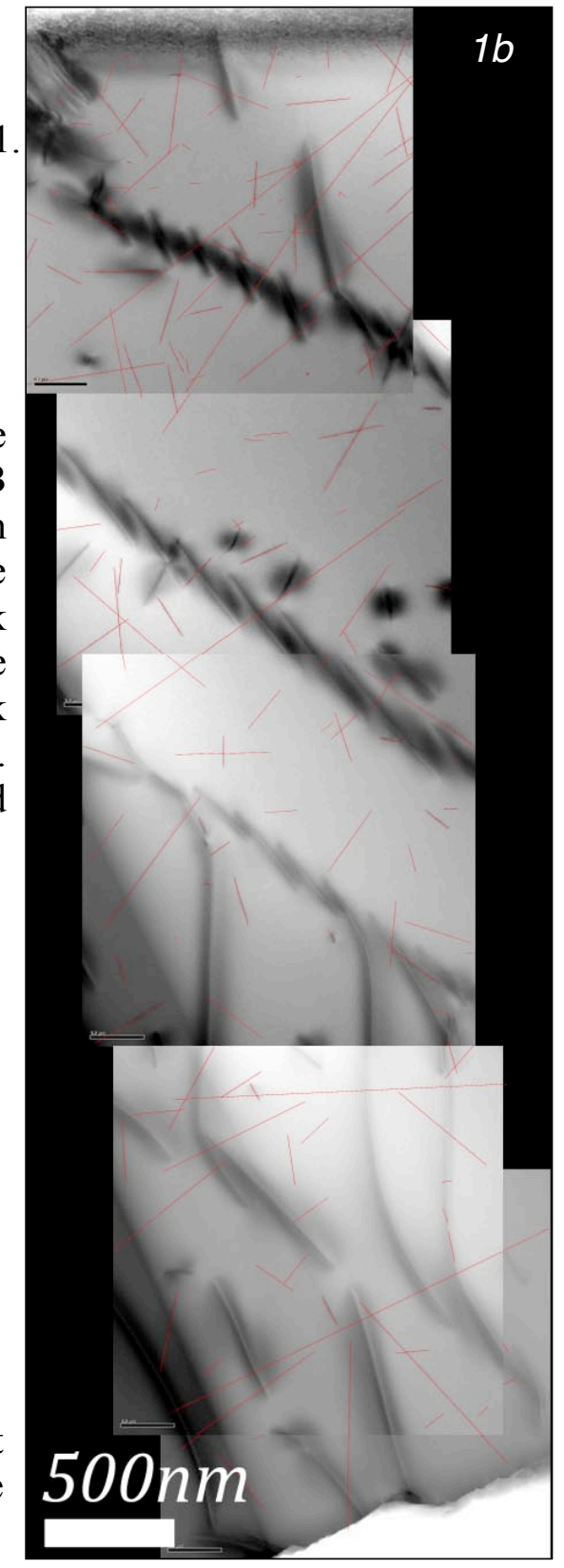

